\title{
Acceleration of Cutaneous Wound Healing by Transient p53 Inhibition
}

\author{
B. Vollmar, A. M. El-Gibaly, C. Scheuer, M. W. Strik, H.-P. Bruch, and \\ M. D. Menger \\ Institute for Clinical and Experimental Surgery (BV, AME-G, CS, MDM), University of Saarland, Homburg/Saar, and \\ Department of Surgery (AME-G, MWS, H-PB), Medical University of Lübeck, Lübeck, Germany
}

SUMMARY: The increase of cell proliferation during early wound healing is thought to be regulated by a decrease of apoptosis. In contrast, the reduction of cellularity during final wound maturation may be controlled by an increase of apoptotic cell death. Herein we studied whether p53 is involved in wound healing-associated apoptosis and whether transient inhibition of p53 is effective to improve the early healing process of cutaneous wounds. Using intravital microscopic and immunohistochemical techniques in hairless mice, we demonstrated that in vivo inhibition of p53 by pifithrin- $\alpha$ (PFT- $\alpha ; 2.2 \mathrm{mg} / \mathrm{kg}$ ip) accelerates early epithelialization and neovascularization of cutaneous wounds by (i) promoting leukocyte recruitment, (ii) increasing cell proliferation, and (iii) reducing apoptotic cell death. We further show that final wound closure with down-regulation of cell proliferation is not inhibited by PFT- $\alpha$ treatment, indicating that transient blockade of p53 function does not affect the process of wound maturation. Western blot analysis revealed that PFT- $\alpha$ lowered nuclear but not cytoplasmic p53, implying that cytoplasmic retention of p53 mediates the antiapoptotic effects of PFT- $\alpha$. Furthermore, PFT- $\alpha$ significantly increased expression of proliferating cell nuclear antigen protein in whole extracts of cutaneous tissue and caused a rise in proliferation of wild-type, but not mutant, p53-expressing keratinocytes. From our study we conclude that transient inhibition of p53 supports the early cell proliferation required for rapid tissue repair and that this may represent an attractive approach in the treatment of delayed wound healing. (Lab Invest 2002, 82:1063-1071).

$A$ poptosis serves as a crucial control mechanism not only for the development of organs during embryogenesis but also for the maintenance of tissue homeostasis in the mature organism. There is mounting evidence that the sequence of cellular events that characterizes healing of cutaneous wounds and other tissue repair processes, is tightly regulated by a distinct temporal pattern of cellular apoptosis (Greenhalgh, 1998). The three classically defined phases of wound healing, ie, inflammation, tissue formation, and tissue remodeling (Singer and Clark, 1999), involve the differential participation of resident cells and infiltrating leukocyte subtypes. After the initial hemostatic event, early wound repair is characterized by the invasion of neutrophils, macrophages, and lymphocytes, which serve (along with immunologic effector cells) as sources of inflammatory and growthpromoting cytokines (Gillitzer and Goebeler, 2001). Then, fibroblasts migrate, proliferate, and synthesize extracellular matrix components, participating in the

DOI: 10.1097/01.LAB.0000024363.37866.45

Received April 22, 2002.

B. Vollmar and A. M. El-Gibaly contributed equally to this work. A. M. El-Gibaly is supported by a grant of the Ministry of Higher Education, Egypt. B. Vollmar is the recipient of a Heisenberg Stipendium of the Deutsche Forschungsgemeinschaft, Bonn-Bad Godesberg, Germany (Vo 450/6-1 and 6-2).

Address reprint requests to: Dr. B. Vollmar, Institute for Clinical and Experimental Surgery, University of Saarland, 66421 Homburg/Saar, Germany.E-mail:exbvol@uniklinik-saarland.de formation of granulation tissue. Cellular infiltration and proliferation must be sufficient and pronounced for normal early progression of repair. It has been discussed that this rapid increase in cell proliferation is allowed by an initial decrease of apoptosis. Later, when the inflammatory process begins to shut down with wound closure and scar evolution, there is a dramatic decrease of cellularity, which has been clearly shown to be mediated by an increase of apoptotic cell death (Desmoulière et al, 1995, 1997). Conversely, when granulation tissue cells are not eliminated because of failing apoptosis, there is pathologic tissue repair and development of hypertrophic scar or keloid, both characterized by a high degree of cellularity (Messadi et al, 1999; Rockwell et al, 1989).

Recently, it has been suggested that the initial decrease of apoptosis during early cell proliferation may be a result of a depression of p53 (Kane and Greenhalgh, 2000). To elucidate this, we herein studied whether p53 is involved in wound healing-associated apoptosis and whether transient inhibition of p53 is effective to improve the initial healing process of cutaneous wounds.

\section{Results}

Tissue Regeneration and Microcirculatory Parameters

Wound surface area amounted to $3.6 \pm 0.3 \mathrm{~mm}^{2}$ (dimethyl sulfoxide; DMSO) and $3.8 \pm 0.1 \mathrm{~mm}^{2}$ (pifithrin- $\alpha$; PFT- $\alpha$ ), respectively. Inhibition of $\mathrm{p} 53$ by 
repetitive application of PFT- $\alpha$ accelerated initial wound epithelialization compared with DMSOtreated controls. Skin defects were found to be epithelialized by $45 \pm 6 \%$ and $97 \pm 1 \%$ at Days 5 and 10 after wounding in the PFT- $\alpha$ group, whereas wound closure in DMSO-treated animals was retarded, with only $25 \pm 8 \%$ and $86 \pm 4 \%$ of the wound surface area covered with epithelium (Fig. $1 \mathrm{~A} ; p<0.05$ versus PFT- $\alpha$ ). At Day 15 , wound repair was terminated in both groups.

The extent of newly vascularized tissue within the wound (area of neovascularization) did not differ between both groups (Day 10: 50\%; Day 15: 80\%) and wound vascularization was almost completed at Day 20 (Fig. 1B). However, after 5 days of tissue repair, wounds of the PFT- $\alpha$-treated animals exhibited higher values of functional capillary density than those of the DMSO-treated animals (Fig. 1C).

During wound healing, the diameter of arterioles and venules in the immediate vicinity of the wound did not markedly differ either between the groups or within each group over time. Moreover, analysis of red blood cell velocity and calculation of volumetric blood flow revealed no statistical differences between the PFT$\alpha$-treated and DMSO-treated animals (Table 1).

\section{Inflammatory Response}

In PFT- $\alpha$-treated animals, leukocytic response upon wounding was more pronounced when compared with that in DMSO-treated animals, as reflected by significantly increased leukocyte adhesion $(p<0.05)$ in capillaries in the immediate vicinity of the wound (Fig. 2A). Distant to the wound, microvascular leukocyte adherence was comparable in wounds of animals of either group (Fig. 2B).

Pearson product moment correlation followed by forward stepwise regression revealed that the dependent variable epithelialization can be predicted from the linear combination of the independent variables neovascularization $(p<0.001 ; r=0.821)$ and microvascular leukocyte adhesion $(p=0.024 ; r=0.842)$ as follows: epithelialization $(\%)=51.969+0.521 \cdot$ neo- vascularization $(\%)+2.453 \cdot$ microvascular leukocyte adhesion. All other variables studied did not significantly add to the ability of the above-mentioned equation to predict epithelialization.

\section{Histology and Immunohistochemistry}

At Day 5, wounds treated with PFT- $\alpha$ revealed higher average scores in cellular invasion and pattern of collagen organization when compared with those of DMSO-treated animals (Table 2). Expression of $\alpha$-smooth muscle actin ( $\alpha$-SMA) was present in all wounds at Day 5 and almost disappeared at Day 20, without marked differences between groups. Moreover, at Day 20, wounds of both DMSO-treated and PFT- $\alpha$-treated animals were well healed, with complete epithelial coverage and advanced collagen organization.

Quantitative analysis of wounds at Day 5 revealed $52.6 \pm 0.8 \%$ of proliferating cell nuclear antigen (PCNA)-positive cells in animals with PFT- $\alpha$ treatment, indicating higher proliferative activity $(\rho<0.01)$ than wounds of DMSO-treated controls, which exhibited only $29.1 \pm 2.0 \%$ PCNA-positive cells (Fig. 3, A and B). Immunostaining of PCNA in wounds at Day 20 showed light staining of epithelial and connective tissue in both DMSO-treated and PFT- $\alpha$-treated animals.

There was definite evidence of apoptosis within the 5-day wounds; however, the degree of apoptosis was significantly $(p=0.032)$ less pronounced in PFT- $\alpha-$ treated animals than in DMSO-treated controls, as given by a score of $2.25 \pm 0.25$ versus $3.75 \pm 0.48$ (Table 2). In hematoxylin- and eosin-stained sections, characteristic morphologic changes, such as cell shrinkage, nuclear and cytoplasmic condensation, nuclear fragmentation, and cellular budding, were identified. The percentage of cells exhibiting these apoptotic features amounted to $9.2 \pm 1.2 \%$ in wounds of DMSO-treated controls but only $5.2 \pm 0.8 \%$ in the wounds with PFT- $\alpha$ treatment $(p=0.033)$.
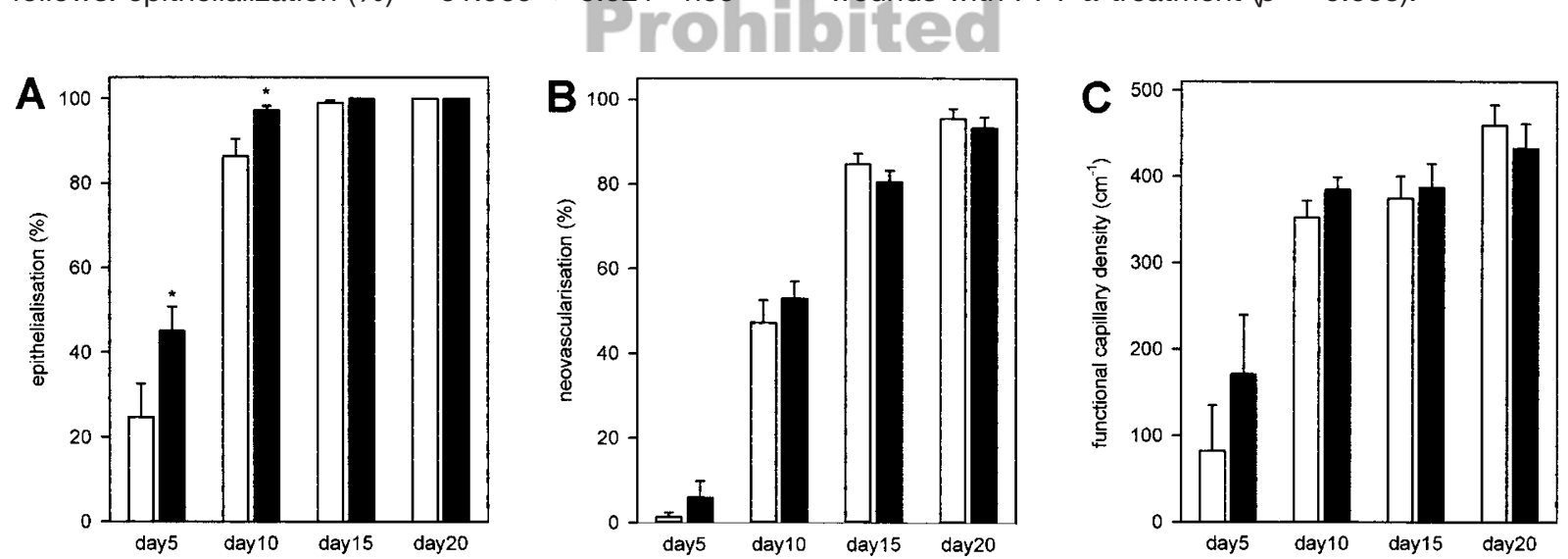

Figure 1.

Epithelialization (\%) (A), neovascularization (\%) (B), and functional capillary density $\left(\mathrm{cm}^{-1}\right)(C)$ of wounds during regeneration at Days $5,10,15$, and 20 , as assessed by intravital fluorescence microscopy. Animals received either pifithrin- $\alpha$ (PFT- $\alpha$; filled bars; $n=5$ ) or dimethyl sulfoxide (DMSO; open bars; $n=5$ ). Values are given as mean \pm SEM. ${ }^{*} p<0.05$ versus DMSO. 
Table 1. Microhemodynamic Parameters in Arterioles and Venules Before Wounding (Day 0 ) and at Days 5, 10, 15, and 20 After Wounding in DMSO-treated and PFT- $\alpha$-treated Animals

\begin{tabular}{lccccc}
\hline & Day 0 & Day 5 & Day 10 & Day 15 & Day 20 \\
\hline Arterioles & & & & & \\
$\quad$ Diameter $(\mu \mathrm{m})$ & & & & & \\
$\quad$ DMSO & $30.5 \pm 1.5$ & $30.7 \pm 2.8$ & $30.6 \pm 0.6$ & $30.7 \pm 1.2$ & $30.7 \pm 1.9$ \\
$\quad$ PFT- $\alpha$ & $30.4 \pm 2.8$ & $32.8 \pm 1.6$ & $30.3 \pm 1.4$ & $30.7 \pm 1.0$ & $32.0 \pm 1.0$ \\
$\quad$ Velocity (mm/sec) & & & & & \\
$\quad$ DMSO & $1.22 \pm 0.07$ & $1.09 \pm 0.15$ & $1.00 \pm 0.15$ & $1.05 \pm 0.07$ & $1.17 \pm 0.13$ \\
$\quad$ PFT- $\alpha$ & $1.19 \pm 0.06$ & $1.12 \pm 0.08$ & $1.05 \pm 0.11$ & $1.00 \pm 0.07$ & $0.98 \pm 0.08$ \\
$\quad$ Volumetric blood flow (nl/sec) & & & & & \\
$\quad$ DMSO & $0.91 \pm 0.12$ & $0.81 \pm 0.15$ & $0.75 \pm 0.13$ & $0.79 \pm 0.10$ & $0.83 \pm 0.08$ \\
$\quad$ PFT- $\alpha$ & $0.87 \pm 0.13$ & $0.95 \pm 0.09$ & $0.78 \pm 0.13$ & $0.75 \pm 0.08$ & $0.78 \pm 0.08$ \\
Venules & & & & & \\
$\quad$ Diameter ( $\mu \mathrm{m})$ & & & & \\
$\quad$ DMSO & $54.6 \pm 2.3$ & $51.5 \pm 2.9$ & $53.1 \pm 4.1$ & $51.4 \pm 3.6$ & $53.5 \pm 3.0$ \\
$\quad$ PFT- $\alpha$ & $54.8 \pm 2.8$ & $56.4 \pm 1.5$ & $53.4 \pm 3.0$ & $52.7 \pm 3.0$ & $54.6 \pm 2.6$ \\
$\quad$ Velocity (mm/sec) & & & & \\
$\quad$ DMSO & $0.50 \pm 0.06$ & $0.56 \pm 0.02$ & $0.56 \pm 0.06$ & $0.52 \pm 0.06$ & $0.57 \pm 0.08$ \\
$\quad$ PFT- $\alpha$ & $0.50 \pm 0.03$ & $0.52 \pm 0.05$ & $0.57 \pm 0.06$ & $0.52 \pm 0.07$ & $0.55 \pm 0.05$ \\
$\quad$ Volumetric blood flow (nl/sec) & & & & \\
$\quad$ DMSO & $1.16 \pm 0.10$ & $1.17 \pm 0.11$ & $1.25 \pm 0.23$ & $1.04 \pm 0.10$ & $1.33 \pm 0.22$ \\
$\quad$ PFT- $\alpha$ & $1.18 \pm 0.10$ & $1.29 \pm 0.11$ & $1.24 \pm 0.13$ & $1.10 \pm 0.13$ & $1.28 \pm 0.14$ \\
\hline
\end{tabular}

DMSO, dimethyl sulfoxide; PFT- $\alpha$, pifithrin- $\alpha$.

Values are given as mean \pm SEM.

\section{Western Blot Analysis of p53 and PCNA}

PFT- $\alpha$ did affect the nuclear to cytoplasmic ratio of the p53 protein inasmuch as PFT- $\alpha$ lowered the levels of nuclear p53 but not of cytoplasmic p53 protein, with a final ratio of 3.4 when compared with the ratio of $5.0 \mathrm{in}$ the DMSO-treated animals (Fig. 4). In line with immunohistochemistry of PCNA in cutaneous wounds, PFT- $\alpha$-treated animals revealed higher expression of PCNA protein in whole skin extracts than DMSOtreated animals (Fig. 3C).

\section{Proliferation Assay}

To further substantiate the hypothesis that PFT- $\alpha$ enhances proliferation via modulation/inhibition of apoptosis in a cutaneous wound healing model, we additionally performed in vitro experiments, eg, the colorimetric indirect proliferation assay WST-1, which is based on the cleavage of the tetrazolium salt WST-1 by mitochondrial dehydrogenases in viable cells, to study the effect of PFT- $\alpha$ on cell proliferation. Although metabolism of the $\mathrm{p} 53 \mathrm{mt} / \mathrm{mt}$ HaCaT cells was not affected by either PFT- $\alpha(20 \mu \mathrm{M})$ or DMSO, wildtype p53-expressing human keratinocyte (HKER) cells showed increased metabolism upon the 24-hour exposure to PFT- $\alpha$ when compared with DMSOexposed cells and unexposed control cells (Fig. 5).

\section{Discussion}

The present in vivo microscopic study in mice communicates the following major findings: (i) Inhibition of p53 by PFT- $\alpha$ caused an acceleration of initial wound epithelialization under noncompromised tissue condi- tions. (ii) PFT- $\alpha$ further led to higher functional capillary density and was associated with increased leukocyte recruitment within the wounds. Notably, the antiapoptotic strategy by PFT- $\alpha$ did not interfere with wound maturation, as indicated histologically by normal solid tissue repair. (iii) Immunohistochemical analysis of PCNA and terminal deoxynucleotidyl transferasemediated dUTP nick-end labeling (TUNEL) staining revealed that enhanced epithelialization within the early tissue repair phase in PFT- $\alpha$-treated animals can best be attributed to high cellular proliferation caused by transient blockade of p53-dependent apoptosis.

\section{Methodologic Remarks}

The hairless mouse ear model is ideally suited for repeated direct quantitative in vivo measurements of microvascular changes occurring during the entire wound healing process (Barker et al, 1989). The fullthickness wound represents a clinically relevant and reproducible model of normal wound healing. The noninvasive microcirculatory observations can be repeatedly performed over time in the same animal and in the same observations fields, reducing the biologic variation to a minimum. The combined study of morphologic and functional changes during wound repair allows for new insights into the mechanisms of healing and objective assessment of the efficiency of novel treatment modalities, such as blockade of p53 by PFT $-\alpha$. One might argue that using healthy organisms, in which wounds heal so rapidly, does not fit with the clinical situation in which many medical and surgical complications can be attributed to wound healing disorders on the basis of atherosclerosis and diabetes 
A inside the wound

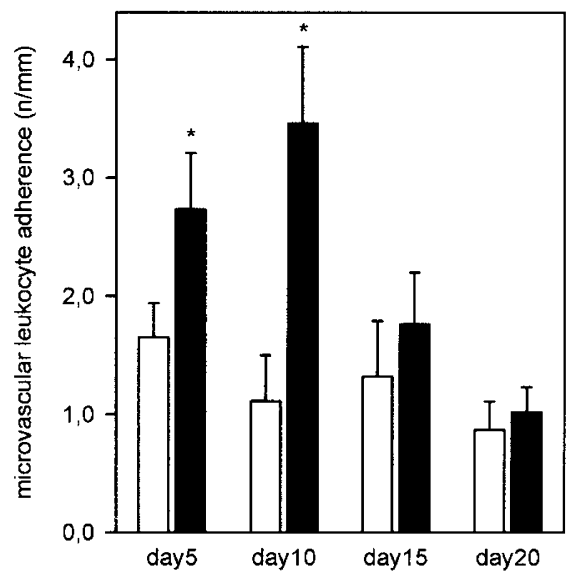

B distant to the wound

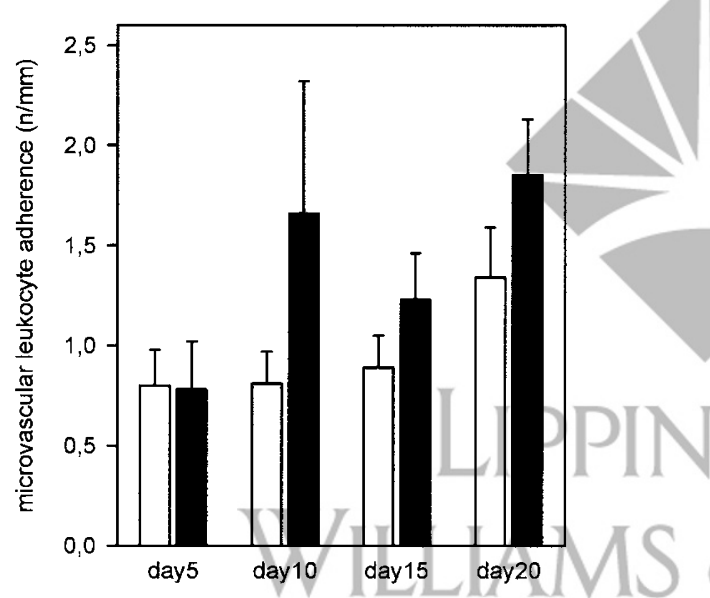

Figure 2.

Microvascular leukocyte adherence $(\mathrm{n} / \mathrm{mm})$ inside the wound $(A)$ and distant to the wound (B) during wound regeneration at Days 5, 10, 15, and 20, as assessed by intravital fluorescence microscopy. Animals received either PFT- $\alpha$ (filled bars; $n=5$ ) or DMSO (open bars; $n=5$ ). Values are given as mean \pm SEM. ${ }^{*} p<0.05$ versus DMSO.

(Greenhalgh et al, 1990). Indeed, changes in healing in uncompromised tissue can be hard to measure; however, interpretation of data does not have to consider multiple unspecific disease-related side effects and results are directly attributable to the drug administered.

As stated by Komarov et al (1999), intraperitoneal application of $2.2 \mathrm{mg} / \mathrm{kg}$ PFT- $\alpha$ failed to exhibit signs of systemic or local toxicity. Besides normal behavior and aspect of the animals, analysis of arterial blood cell counts and cutaneous microcirculatory parameters distant to the wound reflected physiologic conditions (data not shown), as observed in nontreated healthy animals (Barker et al, 1989; Bondár et al, 1991).

\section{Wound Regeneration}

To solve the frequent clinical problem of wound healing failure, a variety of therapeutic regimens for acceleration of wound healing have been investigated in both experimental (Greenhalgh et al, 1990; Roesken et al, 2000) and clinical settings (Brenman, 1991; Cohn, 1986; Jorneskog et al, 1993). Thereby, major efforts in wound healing research have focused on the role of angiogenesis (Hunt et al, 1984), growth factors (Brown et al, 1989; Greenhalgh et al, 1990; Savage et al, 1987), and various mediators (Breslin et al, 1988; Gillitzer and Goebeler, 2001) during the phase of tissue formation. With upcoming and steadily increasing experience on apoptosis, the mechanistic factors that underlie the control of this form of cell death have meanwhile been broadly integrated in the complex biologic network of wound healing (Desmoulière et al, 1997). To our knowledge, however, the present study represents the first attempt to manipulate wound healing by targeting p53-dependent apoptosis.

It is well established that normal tissue repair needs an active proliferative state with granulation tissue formation, which inevitably contributes to the healing of injured tissue. In line with reports of Antoniades et al (1994), demonstrating strong immunostaining with the $\mathrm{Ki}-67$ antibody at the site of cutaneous injury on Days 1 to 3 in swine, we found high proliferative activity by PCNA staining within and in the direct vicinity of the wound during the early phase of tissue repair. It has been reported that this early phase of active cellular proliferation is accompanied by a progressive expression of $\mathrm{c}$-sis/platelet-derived growth factor-B, its receptor $\beta$ (platelet-derived growth factor- $\mathrm{R} \beta$ ), and other mitogenic growth factors (Antoniades et al, 1993, 1994), whereas analysis of both mRNA and protein of the cell cycle arrest inducing p53 gene revealed suppressed expression during that period (Antoniades et al, 1994; Kane et al, 2000). Because tissue repair involves rapid increases in cell proliferation, it makes sense to expect p53 levels to decrease to allow for the sudden need in increase of cellularity.

In supposition of this view, p53 inhibition by PFT- $\alpha$ turned out to be of benefit in the process of wound healing, as indicated in the present study by the accelerated but still controlled wound repair. Treatment with PFT- $\alpha$ increased the rate of cellular infiltration and enhanced epithelial overgrowth of wounds. These in vivo effects presumably result directly or indirectly from the antiapoptotic effect of p53inhibiting PFT- $\alpha$, as strongly underlined by the significantly reduced rate of apoptotic cells within the wounds. This hypothesis is further supported by the fact that PFT- $\alpha$ lowered the protein levels of nuclear but not cytoplasmic p53, implying that cytoplasmic retention of p53 mediates the antiapoptotic effects of PFT- $\alpha$, similarly as has been described for the distribution of p53 in PFT- $\alpha$-treated concanavalin A cells upon ultraviolet irradiation (Komarov et al, 1999). Given the complex and multifaceted nature of the mechanisms by which p53 induces apoptosis (Bates and Vousden, 1999), the modulation of the nuclear to cytoplasmic p53 ratio by PFT- $\alpha$ may not be the only mechanism of antiapoptotic PFT- $\alpha$ action. Enhanced wound healing by PFT- $\alpha$ can further be attributed to the subsequent increase in PCNA expression, as strongly evidenced by immunohistochemistry and 
Table 2. The Effects of PFT- $\alpha$ on Wound Healing Evaluated at Day $\mathbf{5}$ by Using Qualitative Scores for Cellular Invasion, Vascularity, and Collagen Organization (0-3) as well as for Apoptosis (0-5)

\begin{tabular}{ccccc}
\hline & Cellularity & Vascularity & Collagen organization & Apoptosis \\
\hline DMSO & $1.75 \pm 0.25$ & $2.75 \pm 0.25$ & $1.5 \pm 0.29$ & $3.75 \pm 0.48$ \\
PFT- $\alpha$ & $2.50 \pm 0.29$ & $2.75 \pm 0.25$ & $2.50 \pm 0.50$ & $2.25 \pm 0.25^{*}$ \\
\hline
\end{tabular}

Values are given as mean \pm SEM. ${ }^{\star} p<0.05$ vs DMSO.

Western blot protein analysis of cutaneous tissue. PFT$\alpha$-dependent modulation of cell apoptosis/proliferation has further been proven by the fact that exposure to PFT- $\alpha$ caused an expansion in the number of viable wild-type, but not mutant, p53-expressing keratinocytes, with increased overall activity of mitochondrial dehydrogenases and degradation of the tetrazolium salt WST-1 to formazan. As a result, wounds of PFT- $\alpha$-treated animals exhibited a thicker and more cellular granulation tissue than wounds of vehicle (DMSO)-treated animals. The higher leukocytic recruitment in wounds of PFT- $\alpha-$ treated animals may in addition contribute to the enhanced wound repair, because this inflammatory response ensues the microenvironment suitable for tissue reparation (Kainulainen et al, 1998; Rao et al, 1995) and predicts, as given in the present correlation analysis, the extent of epithelialization.

There is general agreement that once an epithelium covers a wound, then inflammation needs to be downregulated and granulation tissue evolves into scar tissue (Martinez-Hernandez, 1988). In situ end labeling of fragmented DNA and detailed electron microscopic evaluation of late phases of wound healing revealed that apoptotic cell death is responsible for the disappearance of cells and the decrease in cellularity during the transition between granulation tissue and scar (Desmoulière et al, 1995). With the assumption that late apoptotic resolution of wound reparation tissue could fail under conditions of p53 blockade, one might expect delayed or impeded wound maturation in PFT- $\alpha$-treated animals. However, $\alpha$-SMA staining, serving as an indicator of smooth muscle cell differentiation, disappeared in wounds of PFT- $\alpha$-treated animals comparably as in DMSO-treated animals. In accordance with Desmoulière et al (1995), this can be interpreted as apoptotic loss of terminally differentiated cells and indicates final maturation of wound tissue.

Thus, without impeding the late wound repair phase that depends on an intact apoptotic cell program, p53-inhibiting PFT- $\alpha$ is of benefit in early wound tissue regeneration, as reflected by the acceleration of the initial epithelialization. Transient inhibition of p53 seems to support early cell proliferation, which is required for rapid tissue repair and might therefore represent an attractive tool in the treatment of delayed wound healing.

\section{Materials and Methods}

\section{Animal Model}

Homozygous (skh-1) hairless mice of both sexes (8-12 weeks old, 22-30 gm body weight) were used in this study. Animals were housed one per cage and had free access to standard rodent laboratory chow and tap water. Room temperature was kept constant

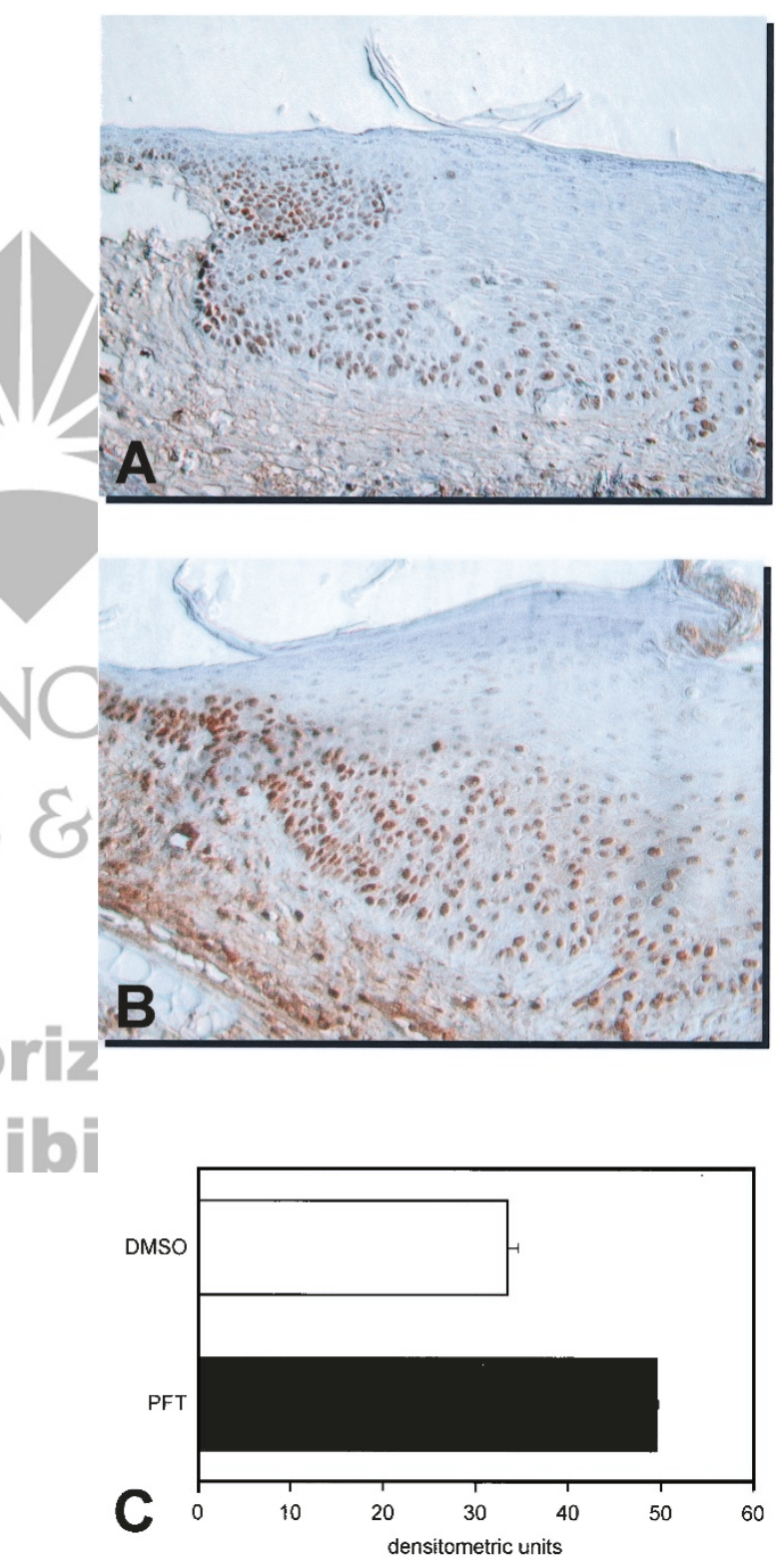

Figure 3.

$\mathrm{A}$ and $\mathrm{B}$, Photomicrographs of 5-day wounds immunohistochemically stained for proliferating cell nuclear antigen (PCNA) in a DMSO-treated (A) and a PFT- $\alpha$-treated animal (B). Note the markedly higher rate of PCNA-positive cells, indicating higher proliferative activity in the PFT- $\alpha-$ treated animal. Magnification, $\times 200$. C, PCNA protein expression, as assessed by Western blot analysis in cutaneous tissue of DMSO-treated and PFT- $\alpha$-treated animals. 
DMSO
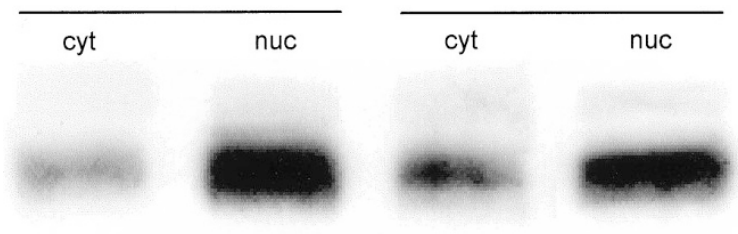

Figure 4.

Representative Western blot displaying the modulation of cytoplasmic (cyt) and nuclear (nuc) distribution of p53 in cutaneous tissue of DMSO-treated and PFT- $\alpha$-treated animals.

at 22 to $24^{\circ} \mathrm{C}$ with a 12-hour dark and light cycle. Upon approval of the experimental protocol by the local animal care committee, the experiments were conducted in accordance with the German legislation on protection of animals and the NIH Guide for the Care and Use of Laboratory Animals (Institute of Laboratory Animal Resources, National Research Council).

Before the surgical intervention of wounding, mice were anesthestized by injection of ketamine/xylazine (90/25 mg/kg ip) and placed prone on a plexiglas stage with the ear to be wounded stretched ventral surface down on the stage. As described in detail previously (Bondár et al, 1991), the selected area on the dorsal skin of the ears was marked with a circular ink stamp ( $2.2 \mathrm{~mm}$ in diameter). Using microscissors and a stereoscopic operating microscope (M650; Wild, Heerbrugg, Switzerland), skin and subcutaneous tissue were excised within the marked area down to the cartilage (depth $\sim 150 \mu \mathrm{m}$ ), thus creating a full dermal thickness wound of approximately $4 \mathrm{~mm}^{2} /$

\section{Experimental Groups}

A total of seven mice received PFT- $\alpha$ (Alexis Biochemicals, San Diego, California) intraperitoneally in a dose of $2.2 \mathrm{mg} / \mathrm{kg}$ body weight (Komarov et al, 1999) at Day -1 before wounding and every second day during the entire observation period of 20 days. Control animals $(n=7)$ were identically treated with equivalent volumes of the vehicle DMSO (Sigma, Deisenhofen, Germany).

\section{Fluorescence Microscopic Analysis of Wound Repair}

By use of a modified fluorescence microscope with a 100W HBO mercury lamp (Axiotech, Zeiss, Jena, Germany), attached to a blue (450-490 nm/>520 nm) and green filter set $(530-560 />580 \mathrm{~nm})$, wound repair was analyzed by the epi-illumination technique and recorded on videotape (S-VHS Panasonic AG 7350, Matsushita, Tokyo, Japan) using a CCD video camera (FK 6990; COHU, Prospective Measurements Inc., San Diego, California). An additional halogen light source (Schott KL1500; Zeiss) allowed for analysis of wounds by the transillumination technique. With the use of long distance objectives $(2.5 \times / 0.075,4 \times / 0.16$, and $10 \times / 0.30$; Zeiss) and a water immersion objective

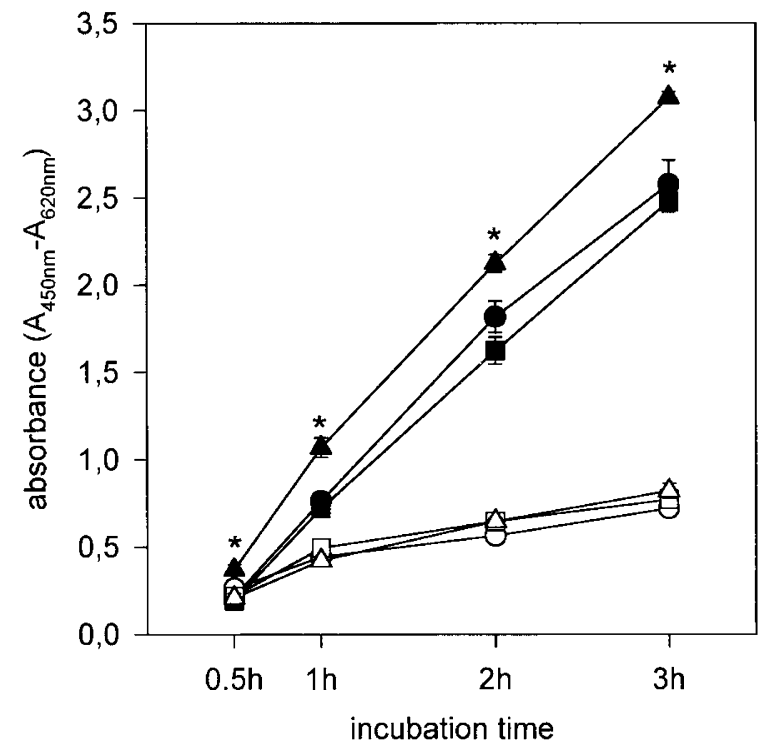

Figure 5.

Kinetics of metabolism of the cell proliferation reagent WST-1 in HaCaT (p53 $\mathrm{mt} / \mathrm{mt}$; open symbols) and human keratinocyte (p53 wt/wt; filled symbols) cells, which were untreated (circles), exposed to DMSO (squares), and exposed to PFT- $\alpha$ (triangles) for 24 hours. At $0.5,1,2$, and 3 hours after WST-1 incubation, the absorbance was determined by an ELISA reader. Mean \pm SEM; * $p<0.05$ versus untreated cells.

(W 20×/0.5; Zeiss), magnifications of $\times 64, \times 91$, $\times 217$, and $\times 491$ were achieved on the video screen (PVM-2130 QM; Sony, Munich, Germany). Contrast enhancement for visualization of the wound $(\times 64$, $\times 91$, and $\times 217$ magnification) and the microcirculatory network ( $\times 491$ magnification) as well as for assessment/ of microcirculatory parameters was achieved using 5\% FITC-labeled dextran $(0.2 \mathrm{ml}$, molecular weight 150,000; Fluka, Buchs, Switzerland). Leukocyte-endothelium interaction was studied after in vivo staining of leukocytes with $1 \%$ rhodamine $6 \mathrm{G}$ (0.2 ml, molecular weight 476; Sigma). The fluorescent tracers were injected intravenously into a tail vein before each microscopic observation time point.

\section{Microcirculatory Parameters}

The analysis of the kinetics of healing included the planimetric evaluation of (i) wound epithelialization and (ii) wound vascularization until complete wound closure (both given in percent of the original wound surface area). Although wound vascularization was assessed by blue light epi-illumination after iv injection of FITC-dextran, epithelialization of wounds was recorded by the trans-illumination technique by monitoring the leading edge of epithelial cell growth across the cartilage bed. The epithelialization was considered complete when the entire wound surface area was covered with cells. Measurements of microcirculatory parameters included the assessment of arteriolar and venular red blood cell velocity $(\mathrm{mm} / \mathrm{sec})$, inner luminal diameter of arterioles and venules $(\mu \mathrm{m})$ in preselected regions of interest, and functional capillary density $\left(\mathrm{cm}^{-1}\right)$ within the wound, at the wound margin, and 
outside the wound. Leukocyte-endothelium interaction was studied within capillaries inside the wound, at the wound margin, and outside the wound (given as number of adherent leukocytes per length of capillaries in the area of interest; $\mathrm{n} / \mathrm{mm}$ ).

Baseline values of the microcirculatory parameters including leukocyte-endothelial cell interaction were determined at Day -1 before wounding. Directly after wounding (Day 0), the wound surface area was assessed by the transillumination technique. Analysis of wound epithelialization and vascularization, microcirculatory parameters, and the leukocyte-endothelial cell interaction was performed at Days 5, 10, 15, and 20 after wounding (five animals per group).

\section{Histology and Immunohistochemistry}

Additional series of experiments were performed for tissue sampling at Day 5 after wounding (two animals per group). Ears at both Days 5 and 20 after wounding were fixed in $10 \%$ phosphate-buffered formalin for 2 to 3 days and embedded in paraffin. From the paraffinembedded tissue blocks, $5-\mu \mathrm{m}$ sections were serially cut and stained with hematoxylin-eosin (HE) for routine histology, with a trichromatic solution for assessment of extracellular matrix deposition (Ladewig staining), and immunohistochemically for demonstration of $\alpha$-SMA and PCNA using indirect immunoperoxidase techniques.

For immunohistochemistry, deparaffinized sections were incubated with $3 \% \mathrm{H}_{2} \mathrm{O}_{2}$ to block endogenous peroxidase. Sections for staining of PCNA were further microwave-heated in citrate buffer $(\mathrm{pH} 6.0)$ at $700 \mathrm{~W}$ for 10 minutes. Mouse anti-human $\alpha$-SMA (1:400; Sigma) and mouse anti-păn PCNA antibodies (1:50; Dako, Hamburg, Germany) were used as primary antibodies, incubated 60 minutes at room temperature. A biotinylated rabbit anti-mouse antibody (Vector Laboratories, Burlingame, California) diluted 1:200 was used as secondary antibody for streptavidin-biotin-complex peroxidase staining (Vectastain ABC-peroxidase-kits; Camona, Wiesbaden, Germany). 3,3' diaminobenzidine was used as chromogen. The sections were counterstained with hematoxylin and examined by light microscopy (Model BX60F; Olympus Optical Company, Tokyo, Japan).

Histologic sections of the wounds were systematically analyzed with respect to cellular invasion and vascularity, using an arbitrary scale from 0 to $3(0=$ none, $1=$ minimal, 2 = moderate, $3=$ maximal). Development of an organized pattern of collagen deposition was estimated also with an arbitrary scale of 0 to 3 , with a score of 3 exhibiting the most advanced collagen organization. Quantitative analysis of PCNA expression is given by the percentage of positive cells in the tissue sections. Mean PCNA values were obtained by counting the number of positive cells in three to four consecutive fields.

For in situ staining of apoptotic cells, the TUNEL method was performed using a commercially available kit (Roche, Mannheim, Germany) according to the manufacturer's instructions. Sections were counter- stained with hemalaun. The degree of apoptosis was estimated with a qualitative scoring system that was based on the overall amount of apoptosis observed in the wound. Zero was assigned for no apoptosis, 1 for minimal, 2 for few, 3 for moderate, 4 for heavy, and 5 for maximal amounts of apoptosis (Brown et al, 1997). In addition, apoptotic cells, ie, cells characterized by cell shrinkage, nuclear and cytoplasmic condensation, nuclear fragmentation, or cellular budding, were assessed in HE-stained sections of the wounds and given in percentage of all cells within an observation field.

\section{Western Blot Analysis}

The procedure used to prepare nuclear and cytoplasmic protein extracts is a modification of the method described by Schreiber et al (1989). After manual homogenization of skin in buffer $\mathrm{A}(10 \mathrm{~mm}$ Tris, $\mathrm{pH} 7.3$, $10 \mathrm{~mm} \mathrm{KCl}, 1.5 \mathrm{~mm} \mathrm{MgCl} 2,0.5 \mathrm{~mm}$ phenylmethylsulfonyl fluoride [PMSF], $0.5 \mathrm{~mm}$ $\beta$-mercaptoethanol) and centrifugation for 25 seconds at $16,000 \times g$ and $4^{\circ} \mathrm{C}$, the pellet was resuspended in lysis buffer (buffer $\mathrm{A}$, containing $0.4 \%$ Nonidet P-40) and kept on ice for 10 minutes. By centrifugation for 5 minutes at $16,000 \times g$ and $4^{\circ} \mathrm{C}$, the supernatant was saved as the cytoplasmic fraction. The nuclei were then resuspended in buffer $\mathrm{C}(20 \mathrm{~mm}$ Hepes, $\mathrm{pH} 7.9$, $400 \mathrm{~mm} \mathrm{NaCl}, 1 \mathrm{~mm}$ EDTA, $1 \mathrm{~mm}$ dithiothreitol, $1 \mathrm{~mm}$ PMSF) and incubated on ice for 15 minutes with occasional shaking. The sample was then centrifuged for 5 minutes at $16,000 \times g$ and $4^{\circ} \mathrm{C}$ to save the supernatant as a nuclear-soluble fraction. For whole protein extracts, skin was homogenized in lysis buffer (10 mm Tris, pH 7.5, $10 \mathrm{~mm}$ NaCl, $0.1 \mathrm{~mm}$ EDTA, 0.5\% Triton $\mathrm{X} 100,0.02 \% \mathrm{NaN}_{3}, 0.2 \mathrm{~mm} \mathrm{PMSF}$ ), incubated for 30 minutes on ice, and centrifuged for 30 minutes at $16,000 \times g$. The supernatant was saved as a whole protein fraction. Before use, all buffers received a protease inhibitor mixture (1:100 v/v; Sigma). Protein concentrations were determined using the Lowry assay with bovine serum albumin as standard (Lowry et al, 1951). Sixty micrograms of protein/lane were separated discontinuously on SDS polyacrylamide gels (10\% SDS-PAGE for both p53 and PCNA) and transferred to a polyvinyldifluoride membrane (Westran; Schleicher \& Schüll, Dassel, Germany). After blockade of nonspecific binding sites, membranes were incubated for 2 hours at room temperature with mouse monoclonal anti-p53 (1:300; BD Biosciences, PharMingen, Heidelberg, Germany) and mouse anti-pan PCNA antibodies (1:200; Dako) followed by peroxidase-conjugated sheep anti-mouse Ig antibodies (1:5000; Amersham Pharmacia Biotech, Freiburg, Germany) as secondary antibodies. The samples were also examined for the presence of a known nuclear (histone $\mathrm{H} 1)$ and cytoplasmic $\left(\mathrm{I}_{\kappa} \mathrm{B} \alpha\right)$ protein $(12 \%$ SDS-PAGE). Antibodies were diluted 1:200 for the anti-histone antibody and 1:200 for the anti-I $\kappa_{K} \mathrm{~B}$ antibody (Santa Cruz Biotechnology, Heidelberg, Germany). Secondary antibody was peroxidase-conjugated 
sheep anti-mouse Ig antibody (1:5000; Amersham Pharmacia Biotech).

Protein expression was visualized by means of luminol enhanced chemiluminescence (ECL; Amersham Pharmacia Biotech) and exposure of the membrane to a blue light-sensitive autoradiography film (Hyperfilm ECL; Amersham, Braunschweig, Germany). Signals were densitometrically assessed (Bio-Rad, Gel-Dokumentationssystem, Munich, Germany) and-in case of whole protein extracts-normalized to the $\beta$-actin signals (mouse monoclonal anti- $\beta$-actin antibody; 1:5000; Sigma) to correct unequal loading.

\section{Proliferation Assay}

The colorimetric indirect proliferation assay WST-1 (Roche), based on the cleavage of the tetrazolium salt WST-1 by mitochondrial dehydrogenases in viable cells, was used to study the effect of PFT- $\alpha$ on cell proliferation. For this purpose, both HKER (p53 wt/wt) and the human keratinocyte cell line HaCaT (p53 $\mathrm{mt} / \mathrm{mt}$ ) were cultured in either keratinocyte growth medium containing $5 \%$ fetal calf serum (Cell Lining, Berlin, Germany) or DMEM containing 10\% FCS (PAA Laboratories, Linz, Austria). According to the manufacturer's instructions, the assay was performed with $3 \times 10^{4}$ cells/well, either exposed to $20 \mu \mathrm{M}$ PFT- $\alpha$ or equivalent volumes of DMSO for 24 hours. After $0.5,1$, 2 , and 3 hours of WST-1 incubation, absorbance of the formazan product was assessed at $450 \mathrm{~nm}$ using a background control as blank and a reference wavelength of $620 \mathrm{~nm}$ (DigiScan 340T Elisa Reader; Asys Hitech, Eugendorf, Austria). The expansion in the number of viable cells results in an increase in the overall activity of mitochondrial dehydrogenases in the sample, which leads to an increase in the amount of formazan dye formed, which directly correlates to the number of metabolically active cells.

\section{Statistics}

Data are presented as mean \pm SEM. After testing for normal distribution using the Kolmogorov-Smirnov test, analysis of variance (two-way repeated measures ANOVA) was performed to test for group, time, and group $\times$ time interactions, followed by appropriate post-hoc comparison tests including correction for multiple test procedures (Tukey test). Pearson product moment correlation was performed, followed by a forward stepwise regression analysis to evaluate significant correlations between the parameters studied. Statistics were performed using the software package SigmaStat version 2.03 (Jandel Corporation, San Rafael, California).

\section{Acknowledgements}

This paper is dedicated to Prof. G. Harbauer in honor of his 75th birthday. The authors thank Ms. Janine Becker for her excellent technical assistance in histology and immunohistochemistry. The HaCaT and HKER cells were kindly provided by Prof. M. Löbrich,
Department of Biophysics, University of Saarland, Homburg/Saar.

\section{References}

Antoniades HN, Galanopoulos T, Neville-Golden J, Kiritsy CP, and Lynch SE (1993). Expression of growth factor and receptor mRNAs in skin epithelial cells following acute cutaneous injury. Am J Pathol 142:1099-1110.

Antoniades HN, Galanopoulos T, Neville-Golden J, Kiritsy C, and Lynch SE (1994). p53 expression during normal tissue regeneration in response to acute cutaneous injury in swine. J Clin Invest 93:2206-2214.

Barker JH, Hammersen F, Bondàr I, Uhl E, Galla TJ, Menger MD, and Messmer K (1989). The hairless mouse ear for the in vivo studies of skin microcirculation. Plast Reconstr Surg 83:948-959.

Bates S and Vousden KH (1999). Mechanisms of p53mediated apoptosis. Cell Mol Life Sci 55:28-37.

Bondár I, Uhl E, Barker JH, Galla TJ, Hammersen F, and Messmer K (1991). A new model for studying microcirculatory changes during dermal wound healing. Res Exp Med 191:379-388.

Brenman SA (1991). Pentoxifylline as adjunctive therapy in leg ulcer management. J Am Osteopath Assoc 91:677-686.

Breslin RJ, Barbul A, Kupper TS, Knud-Hansen JP, Wasserkrug $H L$, and Efron $G$ (1988). Generation of anti-interleukin 2 factor in healing wounds. Ann Surg 123:305-308.

Brown DL, Kao WWY, and Greenhalgh DG (1997). Apoptosis down-regulates inflammation under the advancing epithelial wound edge: Delayed patterns in diabetes and improvement with topical growth factors. Surgery 121:372-380.

Brown GL, Nanney LB, Griffen J, Cramer AB, Yancey JM, Curtsinger LJ III, Holtzin L, Schultz GS, Jurkiewicz MJ, and Lynch JB (1989). Enhancement of wound healing by topical treatment with epidermal growth factor. $N$ Engl $\mathrm{J}$ Med 321:76-79.

Cohn GH (1986). Hyperbaric oxygen therapy: Promoting healing in difficult cases. Postgrad Med 79:89-92.

Desmoulière A, Badid C, Bochaton-Piallat ML, and Gabbiani G (1997). Apoptosis during wound healing, fibrocontractive diseases and vascular wall injury. Int J Biochem Cell Biol 29:19-30.

Desmoulière A, Redard M, Darby I, and Gabbiani G (1995). Apoptosis mediates the decrease in cellularity during the transition between granulation tissue and scar. Am J Pathol 146:56-66.

Gillitzer R and Goebeler M (2001). Chemokines in cutaneous wound healing. J Leukoc Biol 69:513-521.

Greenhalgh DG (1998). The role of apoptosis in wound healing. Int J Biochem Cell Biol 30:1019-1030.

Greenhalgh DG, Sprugel KH, Murray MJ, and Ross R (1990). PDGF and FGF stimulate wound healing in the genetically diabetic mouse. Am J Pathol 136:1235-1246.

Hunt TK, Knighton DR, Thakral KK, Goodson WH III, and Andrews WS (1984). Studies on inflammation and wound healing: Angiogenesis and collagen synthesis stimulated in vivo by resident and activated wound macrophages. Surgery 96:48-54. 
Jorneskog G, Brismar K, and Fagrell B (1993). Low molecular weight heparin seems to improve local capillary circulation and healing of chronic foot ulcers in diabetic patients. Vasa 22:137-141.

Kainulainen V, Wang H, Schick C, and Bernfield M (1998). Syndecans, heparan sulfate proteoglycans, maintain the proteolytic balance of acute wound fluids. J Biol Chem 273: 11563-11569.

Kane CD and Greenhalgh DG (2000). Expression and localization of p53 and bcl-2 in healing wounds in diabetic and nondiabetic mice. Wound Repair Regen 8:45-58.

Komarov PG, Komarova EA, Kondratov RV, Christov-Tselkov K, Conn JS, Chernov MV, and Gudkov AV (1999). A chemical inhibitor of p53 that protects mice from the side effects of cancer therapy. Science 285:1733-1737.

Lowry OH, Rosebrough NJ, Farr AL, and Randall RJ (1951). Protein measurement with the folin phenol reagent. $J$ Biol Chem 193:265-275.

Martinez-Hernandez A (1988). Repair, regeneration and fibrosis: Pathology. In: Rubin E and Farber JL, editors. Philadelphia: JB Lippincott, 66-95.

Messadi DV, Le A, Berg S, Jewett A, Wen Z, Kelly P, and Bertolami CN (1999). Expression of apoptosis-associated genes by human dermal scar fibroblasts. Wound Repair Regen 7:511-517.
Rao CN, Ladin DA, Liu YY, Chilukuri C, Hou ZZ, and Woodley DT (1995). Alpha 1-antitrypsin is degraded and nonfunctional in chronic wounds but intact and functional in acute wounds: The inhibitor protects fibronectin from degradation by chronic wound fluid enzymes. J Invest Dermatol 105:572-578.

Rockwell WB, Cohen IK, and Ehrlich HP (1989). Keloids and hypertrophic scars: A comprehensive review. Plast Reconstr Surg 84:827-837.

Roesken F, Uhl E, Curri SB, Menger MD, and Messmer K (2000). Acceleration of wound healing by topical drug delivery via liposomes. Langenbeck's Arch Surg 385:42-49.

Savage K, Siebert E, and Swann D (1987). The effect of platelet-derived growth factor on cell division and glycosaminoglycan synthesis by skin and scar fibroblasts. J Invest Dermatol 89:93-99.

Schreiber E, Matthias P, Muller MM, and Schaffner W (1989). Rapid detection of octamer binding proteins with 'miniextracts', prepared from a small number of cells. Nucleic Acids Res 17:6419.

Singer AJ and Clark RAF (1999). Cutaneous wound healing. N Engl J Med 341:738-746.
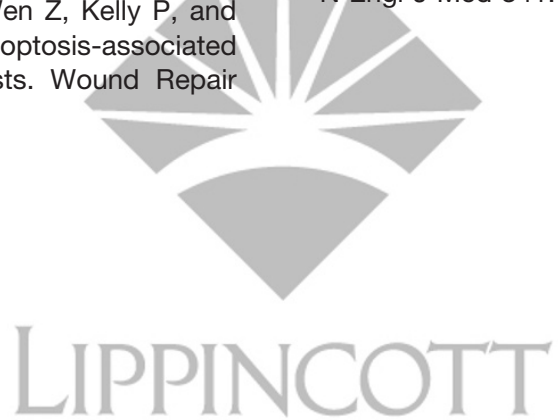

WILLIAMS \& WILKINS

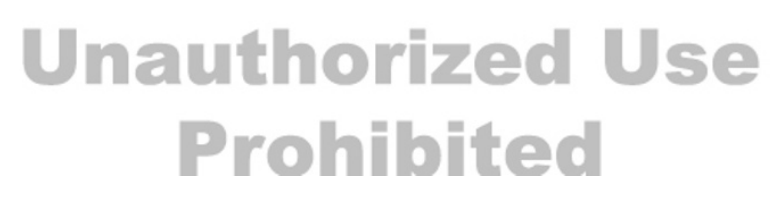

